\title{
Erratum to: 86 successful births and 9 ongoing pregnancies worldwide in women transplanted with frozen-thawed ovarian tissue: focus on birth and perinatal outcome in $\mathbf{4 0}$ of these children
}

\author{
Annette Klüver Jensen ${ }^{1} \cdot$ Kirsten Tryde Macklon ${ }^{2} \cdot$ Jens Fedder $^{3} \cdot$ Erik Ernst $^{4}$. \\ Peter Humaidan ${ }^{5}$ Claus Yding Andersen ${ }^{1}$
}

Published online: 24 January 2017

(C) Springer Science+Business Media New York 2017

Erratum to: J Assist Reprod Genet (2016)

DOI 10.1007/s10815-016-0843-9

The original version of this article unfortunately contained errors in the Discussion section as follows:

1) The whole paragraph starting "It is currently impossible to obtain a complete..." should be replaced with the below paragraph:

It is difficult to obtain a complete picture of this procedure's success rate due to the absence of reports of non- pregnancies in the peer-reviewed papers [43]. The Danish experience is that the success rate of having a positive pregnancy test among the Danish women, who had a pregnancy wish, was $63 \%$. Furthermore, $31 \%$ of the women with a pregnancy wish succeed in conceiving at least one child and $50 \%$ of the pregnancies were naturally conceived [8].

2) The last paragraph of the Discussion section should be removed.

The original article was corrected.

The online version of the original article can be found at http://dx.doi.org/ 10.1007/s10815-016-0843-9.

Annette Klüver Jensen

annetteklueverjensen@gmail.com

1 The Laboratory of Reproductive Biology, Section 5712, Juliane Marie Centre for Women, Children and Reproduction, University Hospital of Copenhagen, University of Copenhagen, Blegdamsvej 9, Rigshospitalet, DK-2100 Copenhagen, Denmark

2 The Fertility Clinic, The JulianeMarie Centre, University Hospital of Copenhagen, Rigshospitalet, Copenhagen, Denmark

3 The Fertility Clinic, University Hospital of Odense, Odense, Denmark

4 The Fertility Clinic, University Hospital of Aarhus, Skejby, Aarhus, Denmark

5 The Fertility Clinic, Skive Regional Hospital, Skive, Denmark and Faculty of Health, Aarhus University, Aarhus, Denmark 